\section{Management of COVID-19-related immune thrombocytopenia by rhTPO}

TO THE EDITOR: Immune thrombocytopenia (ITP), an acquired thrombocytopenia, is defined as a platelet count of $<100,000 / \mu \mathrm{L}$ and is caused by immune-mediated destruction of platelets. The incidence of primary ITP is 3.3 per 100,000 adults per year, with a prevalence of 9.5 per 100,000 adults [1]. ITP is not a single disease, but a thrombocytopenic syndrome that may be primary or secondary to a possible infection or immune process. ITP can be caused by infection with Helicobacter pylori, cytomegalovirus, varicella-zoster virus, hepatitis $C$ virus, and human immunodeficiency virus [2]. Recently, coronavirus infection-related ITP has also been reported [3]. At the end of 2019, a novel coronavirus, designated as severe acute respiratory syndrome coronavirus 2 (SARS-CoV-2), emerged in Wuhan, China, and soon spread to China and other countries [4]. Thrombocytopenia was also observed in different proportions (5-17\%) among patients with coronavirus disease (COVID-19) [5]. Moreover, a case of COVID-19-related ITP has been reported [6]. However, there are no reports of COVID-19-related ITP cases treated with recombinant human thrombopoietin (rhTPO). Here, we have reported a case of a COVID19-related ITP that was successfully treated with rhTPO.

\section{Case report}

A 58-year-old women was admitted due to fever of 20 days and was previously diagnosed with COVID-19 16 days prior. The patient developed fever on January 30, 2020, with occasional cough, shortness of breath, and other discomfort, but with no chest tightness. Chest computed tomography (CT) performed at a local hospital on January 31, 2020 revealed sporadic infectious lesions in both the lungs. The SARS-CoV-2 nucleic acid test results were positive on February 3, 2020. Later, the patient was admitted to the square cabin hospital and received symptomatic supportive treatment, such as antiviral drugs. During the hospital stay, the patient's body temperature returned to normal, and her cough gradually improved. A day before transfer to our hospital, the patient experienced dizziness with emesis once after taking a bath. The peripheral complete blood count test revealed thrombocytopenia, with a peripheral blood platelet count of $25,000 / \mu \mathrm{L}$. The patient's dizziness, nausea, and vomiting did not recur, and no skin or mucosal bleeding was found. The patient was then transferred to our hospital for further treatment.

This patient was in good health, except for a history of hypertension for $>10$ years. She had no history of thrombocytopenia or allergies. She had no history of unexplained recurring skin rashes, joint swelling and pain, dry mouth, or dry eyes. Physical examination revealed unremarkable findings. On admission, the patient was initially diagnosed with COVID-19 and thrombocytopenia. Because the patient had no previous history of thrombocytopenia or autoimmune diseases, ITP after a viral infection was first considered. Laboratory findings were as follows: white blood cell count, 5,450/ $\mu \mathrm{L}$; hemoglobin level, $117 \mathrm{~g} / \mathrm{L}$; platelet count, $15,000 / \mu \mathrm{L}$; C-reactive protein level, within normal range; globulin level, $24.9 \mathrm{~g} / \mathrm{L}$; creatinine level, $33.6 \mathrm{mmol} / \mathrm{L}$; prothrombin time, $14 \mathrm{~s}$; activated partial thromboplastin time, $31 \mathrm{~s}$; D-dimer level, $0.46 \mathrm{mg} / \mathrm{L}$; antinuclear antibody, $<1: 40$. Peripheral blood smear did not reveal any significant abnormalities. Abdominal B-ultrasound did not reveal an enlarged spleen. Because bone marrow aspiration was temporarily unavailable during the special period of the COVID-19 epidemic, she was diagnosed with ITP based on her medical history.

According to the current World Health Organization guidelines [7], corticosteroids should not be used to treat SARS-CoV-2-induced lung injury or shock, except in the setting of a clinical trial. According to the expert consensus statement on the use of corticosteroids in COVID-19 pneumonia [8], corticosteroids are not recommended for patients with mild disease. In addition, because glucocorticoid use suppresses immune function and, thus, prolongs the course of COVID-19, glucocorticoid treatment was not administered. Since the patient's platelet count was $<20,000 / \mu \mathrm{L}$ after admission, which satisfied the standard for emergency treatment, we administered $20 \mathrm{~g}$ of gamma globulin by intravenous infusion (IVIg) combined with rhTPO 15,000 U daily subcutaneously. The patient also received $10 \mathrm{U}$ of platelet transfusion. Two days later, her platelet count was $43,000 / \mu \mathrm{L}$. The patient's platelet count returned to $53,000 / \mu \mathrm{L}$ five days after treatment initiation and was maintained at this level for the next week (Fig. 1). During this period, the patient did not develop fever and did not experience symptoms such as chest tightness or shortness of breath. The SARS-CoV-2 nucleic acid tests that were performed $>24 \mathrm{~h}$ apart revealed negative findings, but showed SARS-CoV-2 immunoglobulin $\mathrm{M}$ positivity. A review of the chest CT showed that the infectious lesions scattered in both the lungs were clearly absorbed. The patient was subsequently discharged and continued to receive rhTPO treatment twice a week. The patient had no cutaneous mucosal bleeding events during hospitalization. The platelet count returned to normal levels after 4 weeks of rhTPO therapy and remained normal after discontinuation of rhTPO.

To our knowledge, there are only a few cases of COVID-19-related ITP. As COVID-19 is a novel disease, treatment of various comorbidities including ITP related to this disease also requires the accumulation of new experience. The treatment experience in this case may have a certain significance for the treatment of similar cases in the future.

\section{Discussion}

Diagnosis of ITP is generally confirmed by reviewing 


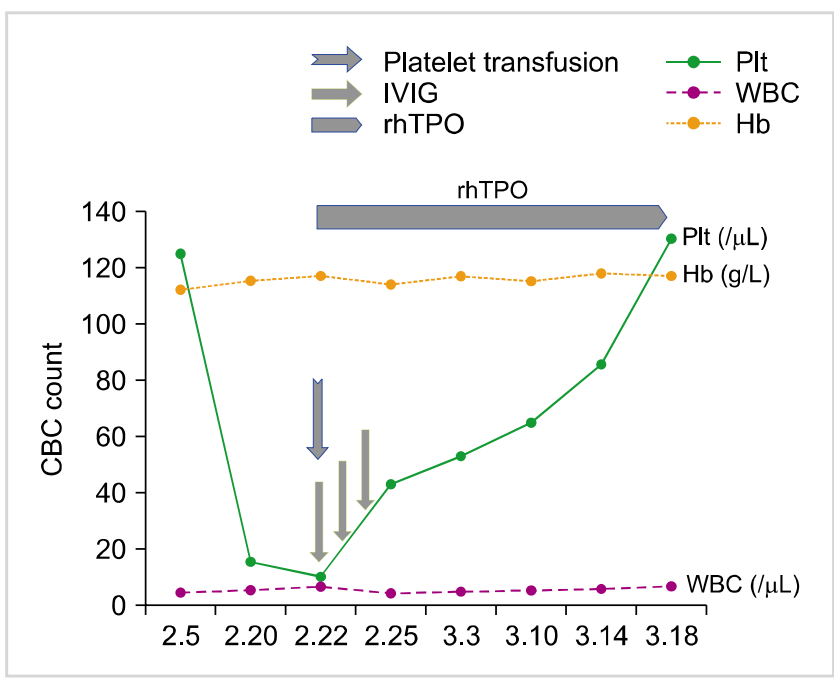

Fig. 1. Dynamic profile of the complete blood count during hospitalization.

Abbreviations: CBC, complete blood count; $\mathrm{Hb}$, hemoglobin; IVIg, gamma globulin by intravenous infusion; Plt, platelet; rhTPO, recombinant human thrombopoietin.

the peripheral smear findings, evaluating the patient's history, and performing physical examination. The patient had no previous history of thrombocytopenia, and thrombocytopenia was not observed early in the diagnosis of COVID-19. Peripheral blood thrombocytopenia was detected 2 weeks after the diagnosis of COVID-19. Laboratory tests ruled out the possibility of other hematological diseases and immune abnormalities leading to thrombocytopenia. The patient did not undergo bone marrow examination because of the COVID-19 epidemic. The ITP International Working Group (IWG) recommends bone marrow examination in patients with new-onset ITP over the age of 60 years. However, the American Society of Hematology guidelines suggest that bone marrow examination may not be necessary for the diagnosis of ITP in any patient population, as supported by some population studies [8]. Therefore, although a bone marrow examination was not performed, the diagnosis of ITP in this patient was valid.

The pathogenesis of ITP involves the loss of tolerance to glycoproteins expressed on platelets and megakaryocytes [9]. Loss of peripheral tolerance caused by infectious diseases, including COVID-19, may result in the development of ITP. Hematological changes in patients with severe acute respiratory syndrome are also common and notably include lymphopenia and thrombocytopenia. Although the development of autoimmune antibodies or immune complexes triggered by viral infection may play a significant role in inducing thrombocytopenia, severe acute respiratory syndrome coronavirus (SARS-CoV) may also directly infect hematopoietic stem/progenitor cells, megakaryocytes, and platelets, inducing their growth inhibition and apoptosis [10]. SARS-CoV-2, which is highly homologous to SARS-CoV, is likely to have similar mechanisms leading to thrombocytopenia. This requires further research in the future.
ITP treatment can be adjusted according to the patient's platelet level and the risk of bleeding and is generally divided into emergency treatment, first-line treatment, and second-line and above treatment. Patients with severe thrombocytopenia or significant bleeding symptoms require hospitalization and emergency treatment to rapidly increase platelet counts. IVIg increases the platelet count in $60-80 \%$ treated patients, often within days, and is effective in patients who have or have not undergone splenectomy, although responses are usually of short duration (1-3 wk) [2]. Platelet transfusion can transiently and rapidly increase platelet count in emergency situations, and concurrent infusion of IVIg may prolong the survival of transfused platelets in some patients. The patient's platelet count in our case was $<20,000 / \mu \mathrm{L}$, and the risk of bleeding was high; therefore, we administered IVIg as emergency treatment and platelet transfusion, which rapidly increased the platelet count.

Glucocorticoids have been the standard first-line treatment for ITP for many years. However, recent studies have found that the combination of multiple treatment methods, such as glucocorticoids, rituximab, and thrombopoietin receptor agonists (TPO-RA), may increase the response rate of ITP treatment. In the last decade, TPO-RA-based megakaryocyte-targeted therapies have shown good results in improving platelet counts in the treatment of ITP [11]. The currently available TPO-RA drugs include romiplostim, eltrombopag, and rhTPO. The efficacy of rhTPO in treating ITP in China has been confirmed in multiple studies [1214]. Because our patient was diagnosed with COVID-19, glucocorticoid-based immunosuppressive therapy was not suitable. Therefore, although IVIg combined with platelet transfusion was used for emergency treatment, we chose rhTPO treatment instead of glucocorticoid treatment for our patient. The patient's platelet count improved and stabilized after receiving this treatment. The patient's treatment effect can be assessed as a response based on the IWG efficacy criteria.

\section{Conclusion}

We have reported a case of a COVID-19-related ITP that improved after treatment with IVIg and rhTPO. As COVID-19, a novel infectious disease, is spreading globally, similar cases may continue to surface. Our patient's medical management can be used as a reference for the treatment of patients with COVID-19 and similar diseases combined with ITP. The diagnosis and treatment of ITP in our patient were performed based on experience during a special period. Accumulation of more cases is warranted for a more comprehensive knowledge of similar diseases. In the future, the detailed mechanism of COVID-19-induced ITP and the best treatment strategy needs further investigation. Relevant research will also increase our comprehensive understanding of the pathogenesis and treatment strategies of ITP. 
Xuzhao Zhang ${ }^{1,2}$, Min $\mathrm{Li}^{2,3}$, Tao Chen ${ }^{2,3}$, Dong $\mathrm{Lv}^{2,4}$, Pengfei Xia ${ }^{2,5}$, Wenbin Qian ${ }^{1}$ ${ }^{1}$ Department of Hematology, ${ }^{2}$ Aid Hubei Medical Team, ${ }^{3}$ Neurology Intensive Care Unit, The Second Affiliated Hospital of Zhejiang University School of Medicine, Hangzhou, ${ }^{4}$ Department of Respiratory Medicine, Shangyu People's Hospital, Shangyu, ${ }^{5}$ Department of Respiratory Medicine, Lanxi People's Hospital, Lanxi, China

Correspondence to: Xuzhao Zhang Department of Hematology, The Second Affiliated Hospital of Zhejiang University School of Medicine, No.88, Jiefang Road. Hangzhou, Zhejiang 310009, China E-mail: zxzzju@zju.edu.cn

Received on Mar. 18, 2020; Revised on Jul. 13, 2021; Accepted on Jul. 14, 2021 https://doi.org/10.5045/br.2021.2020049

\section{Source of support}

This study was supported by a grant from Zhejiang Provincial Natural Science Foundation of China (LY14H 160032 to Xuzhao Zhang) and a grant from Leukemia Research Innovative Team of Zhejiang Province (2011 R50015 to Xuzhao Zhang).

\section{Acknowledgments}

We thank all members of the Aid Hubei Medical Team from the Second Affiliated Hospital of Zhejiang University School of Medicine for their solidarity and cooperation. We also thank all sectors of the society for their efforts in fighting the COVID-19 epidemic.

\section{Authors' Disclosures of Potential Conflicts of Interest}

No potential conflicts of interest relevant to this article were reported.

\section{REFERENCES}

1. Lambert MP, Gernsheimer TB. Clinical updates in adult immune thrombocytopenia. Blood 2017;129:2829-35.

2. Kistangari G, McCrae KR. Immune thrombocytopenia. Hematol Oncol Clin North Am 2013;27:495-520.
3. Magdi M, Rahil A. Severe immune thrombocytopenia complicated by intracerebral haemorrhage associated with coronavirus infection: a case report and literature review. Eur J Case Rep Intern Med 2019;6:001155.

4. Munster VJ, Koopmans M, van Doremalen N, van Riel D, de Wit E. A novel coronavirus emerging in China - key questions for impact assessment. N Engl J Med 2020;382:692-4.

5. Lippi G, Plebani M. Laboratory abnormalities in patients with COVID-2019 infection. Clin Chem Lab Med 2020;58:1131-4.

6. Zulfiqar AA, Lorenzo-Villalba N, Hassler P, Andrès E. Immune thrombocytopenic purpura in a patient with Covid-19. N Engl J Med 2020;382:e43.

7. World Health Organization. Clinical management of severe acute respiratory infection when novel coronavirus (nCov) infection is suspected. Geneva, Switzerland: World Health Organization, 2020. (Accessed February 9, 2020, at https:// www.who.int/publications/i/item/10665-332299).

8. Zhao JP, Hu Y, Du RH, et al. Expert consensus on the use of corticosteroid in patients with 2019-nCoV pneumonia. Zhonghua Jie He He Hu Xi Za Zhi 2020;43:E007.

9. Purohit A, Aggarwal M, Singh PK, et al. Re-evaluation of need for bone marrow examination in patients with isolated thrombocytopenia contributors. Indian J Hematol Blood Transfus 2016;32:193-6.

10. Stasi R. Immune thrombocytopenia: pathophysiologic and clinical update. Semin Thromb Hemost 2012;38:454-62.

11. Yang M, Ng MH, Li CK. Thrombocytopenia in patients with severe acute respiratory syndrome (review). Hematology 2005; 10:101-5.

12. Ghanima W, Cooper N, Rodeghiero F, Godeau B, Bussel JB. Thrombopoietin receptor agonists: ten years later. Haematologica 2019;104:1112-23.

13. Wang S, Yang R, Zou P, et al. A multicenter randomized controlled trial of recombinant human thrombopoietin treatment in patients with primary immune thrombocytopenia. Int J Hematol 2012;96:222-8.

14. Zhou $\mathrm{H}, \mathrm{Xu}$ M, Qin $\mathrm{P}$, et al. A multicenter randomized open-label study of rituximab plus rhTPO vs rituximab in corticosteroid-resistant or relapsed ITP. Blood 2015;125:1541-7. 